\title{
Enantioseparation of Tedizolid phosphate by RP-HPLC, using $\beta$-Cyclodextrin as a Chiral Mobile Phase Additive
}

\author{
Ajit Anerao*, Vikram Dighe, Satish John, Nitin Pradhan \\ R\&D centre (API), Wanbury Ltd., EL-16, TTC Industrial Estate, Mahape, Navi Mumbai 400710, India.
}

\section{ARTICLE INFO}

Article history:

Received on: 22/07/2017

Accepted on: 01/10/2017

Available online: 30/10/2017

Key words:

Tedizolidphosphate; enantiomer; cyclodextrin; method development

\begin{abstract}
Objective: Enantiomeric separation and quantification of S-enantiomer of Tedizolid phosphate drug substance. Material and method: A simple and sensitive reversed phase high performance liquid chromatography (RPHPLC) method is developed by using $\beta$-cyclodextrin $(\beta$-CD) as a chiral mobile phase additive. Effect of the $\mathrm{pH}$ value of aqueous buffer, concentration of chiral additive, composition of mobile phase, and column temperature on the enantioseparation of Tedizolid phosphate was investigated on the Phenomenex Luna, Phenyl-Hexyl, 250 $\mathrm{x} 4.6 \mathrm{~mm}, 5 \mu \mathrm{m}$ HPLC column. A satisfactory resolution was achieved at column temperature $20^{\circ} \mathrm{C}$ using a mobile phase consisting of a mixture of aqueous buffer of $\mathrm{pH} 7.0$ of disodium hydrogen phosphate with additive $\beta$-cyclodextrin, triethylamine and acetonitrile. This analytical method was evaluated by performing method validation as per ICH guideline.

Results: The calibration curve was plotted within the concentration range between 0.30 and $2.25 \mu \mathrm{g} \mathrm{mL}^{-1}$ and the recoveries between 96.9 and $105.3 \%$ were obtained, with regression coefficient $\mathrm{R}^{2}$ is 0.998 . The limit of detection (LOD) and limit of quantitation (LOQ) of S- enantiomer of Tedizolid phosphate is 0.10 and $0.30 \mu \mathrm{g}$ $\mathrm{mL}^{-1}$

Conclusion: The developed method was demonstrated to be accurate, robust and sensitive for the determination of enantiomeric purity of Tedizolid phosphate drug substance.
\end{abstract}

\section{INTRODUCTION}

The chemical name of Tedizolid phosphate is [(5R)-(3\{3-Fluoro-4-[6-(2-methyl-2H-tetrazol-5-yl)pyridin-3-yl]phenyl $\}$ 2-oxooxazolidin-5-yl]methyl hydrogen phosphate. It has an empirical formula of $\mathrm{C}_{17} \mathrm{H}_{16} \mathrm{FN}_{6} \mathrm{O}_{6} \mathrm{P}$ and a molecular weight of $450.32 \mathrm{~g} \mathrm{~mole}^{-1}$ (FDA, 2014). Tedizolid phosphate is a novel oxazolidinone prodrug antibiotic that is converted in vivo by phosphatases to the microbiologically active moiety tedizolid. It has been developed for both oral and intravenous (i. v.) use. Sivextro tablets and lyophilized powder for injection are indicated for the treatment of acute bacterial skin and skin structure infections (ABSSSI) caused by susceptible isolates.

\footnotetext{
* Corresponding Author

E-mail: ajit.anerao@wanbury.com; Mobile+917506734840

Telephone +91 2264570555; Fax +91 2264570553
}

Tedizolid phosphate has one asymmetric center, leading to two possible enantiomers. The absolute configuration at the 5position of the oxazolidinone ring is the $\mathrm{R}$ optical isomer. In vitro stability data, showing high stability of the R-isomer, also support the assumption of a low risk for in vivo inter-conversion in humans (Elaine and Saba, 2014; EMA, 2015). The US Food and Drug Administration and other regulatory agencies have made it mandatory for the manufacturers to investigate each enantiomer of the chiral drug individually (Rauws and Groen, 1994). According to the International Conference on Harmonization (ICH) guidelines, chiral identity, enantiomeric impurity and chiral assay tests may be needed in drug substance and product specifications (Branch, 2005).

Several research papers have been reported in the literature related to quantification of tedizolid phosphate in human plasma (Santini et al., 2015) and its absorption, distribution, metabolism and excretion (Voon et al., 2014). 
In order to quantitatively determine the enantiomeric composition of tedizolid phosphate, very few analytical methods have been reported. These papers were limited to the chiral purity using capillary electrophoresis (Katarzyna et al., 2016). Because of unavailability of simple, cost effective technique, it is prompted to develop method of analysis which can be performed in any quality control laboratory.

During method development different chiral stationary phases were used along with combination with mixture of polar and non-polar solvents. Normal phase as well as reverse phase chiral chromatography with mobilized and immobilized stationary phases were tried but could not achieve satisfactory resolution. In present research $\beta$-cyclodextrin was added in the mobile phase and achieved base to base separation between $\mathrm{R}$ and $\mathrm{S}$ isomer. Instead of using chiral stationary phases, addition of a chiral additive into the mobile phase form a pair of transient diastereomeric complexes, followed by chromatographic separation on an achiral column. Introducing chiral additives in mobile phase has a number of advantages over using chiral stationary phases (Christodoulou, 2010; Płotka et al., 2011).

First, the proposed method is less expensive because achiral column is used which is less expensive as compared to chiral columns. In general, chiral columns are of high price, less stability, low capacity and have a relatively short lifetime. Secondly, this method offers greater flexibility. Diverse achiral columns and chiral additives can be used. Moreover, the approach based on chiral mobile phases can work in both a normal phase and a reversed phase operation mode.

Cyclodextrins separate the enantiomers utilizing the phenomenon of host guest complexation. The affinity of the analyte for cyclodextrin is due to the hydrophobic interaction between the analyte and the cyclodextrin cavity and the hydrogen bonding between the analyte and the functional groups on the cyclodextrin ring.

Cyclodextrin and its derivatives (Han, 1997; Bressolle et al., 1996) have been recognized as the most prevalent chiral mobile phase additives due to the nature of being nontoxic, nonflammable, non-volatile, and stable over a wide range of $\mathrm{pH}$ and negligible absorption in the UV ranges broadly used in chromatographic detection.

They, therefore, have been successfully applied in the enantiomeric separation of a variety of racemates reported in a review article (Bressolle et al., 1996), and recently, of mandelic acid derivatives (Tong et al., 2014), pantoprazole (Guan et al., 2008), cathinone and amphetamine derivatives (Taschwer et al., 2014), phenyllactic acid (Xu et al., 2013), 2-arylpropionic acid (Ye et al., 2010), sertraline (Reyes-Reyes et al., 2014), betacarboline alkaloids (Le'On et al., 2008), and amlodipine (Xie et al., 2014). The proposed analytical method is validated as per International conference on harmonization guidelines ICH Q2-R1 (ICH, 2005) and USFDA (FDA, 2009).

Structure of R-Tedizolid phosphate, S-Tedizolid phosphate and $\beta$-cyclodextrin are given in figure I to III.

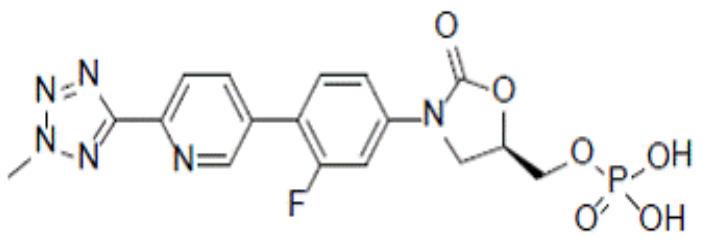

Fig. I: R-Tedizolid phosphate.

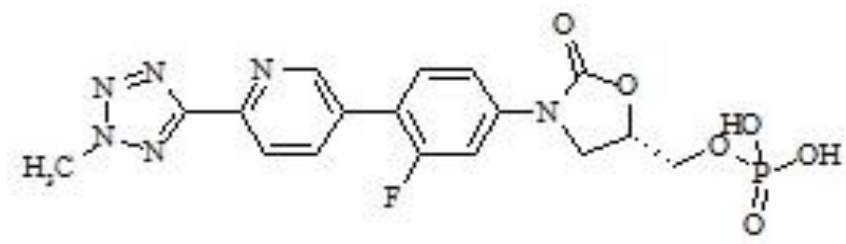

Fig. II: S-Tedizolid phosphate.

A

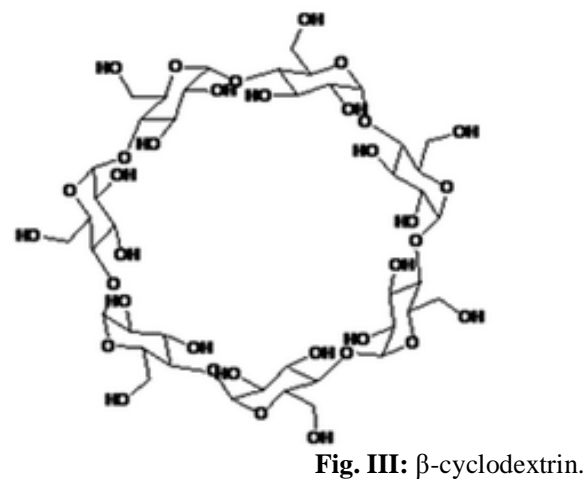

MATERIALS AND METHODS

\section{Chemical and reagents}

$\beta$-cyclodextrin CAS No.7585-39-9 was purchased from Spectrochem, India. R-Tedizolid phosphate and S-enantiomer is synthesized and characterized at Wanbary R\&D center. All HPLC grade chemicals required for mobile phase and sample preparation, acetonitrile, triethylamine, disodium hydrogen phosphate and ophosphoric acid were purchased from Merck-India. HPLC grade N,N-dimethylsulfoxide from Rankem and Siemens Labostar water purification system was used for HPLC grade water to prepare the mobile phase and diluents. Mobile phase was filtered through 0.45 $\mu \mathrm{m}$ nylon filter before use.

\section{Instrumentation}

Shimadzu HPLC system LC-2010 CHT with UV detector and LC solutions software or its equivalent was used. The analysis was carried out on Phenomenex Luna Phenyl-Hexyl, 250 $\mathrm{x} 4.6 \mathrm{~mm}, 5 \mu \mathrm{m}$ Part No.00G-4257-E0 with column temperature is $20^{\circ} \mathrm{C}$. Separation was achieved with gradient elution of mobile phase-A and mobile phase-B with timed programme $\mathrm{T}_{\min } / \mathrm{A}: \mathrm{B}$ : $\mathrm{T}_{0} / 100: 00 ; \quad \mathrm{T}_{50} / 100: 00 ; \quad \mathrm{T}_{55} / 00: 100 ; \quad \mathrm{T}_{65} / 00: 100 ; \quad \mathrm{T}_{66} / 100: 00 ;$ $\mathrm{T}_{75} / 100: 00$. The flow rate was $1.0 \mathrm{~mL} / \mathrm{min}$ and sample injection volume was $5 \mu \mathrm{L}$. Detector wavelength is $300 \mathrm{~nm}$. 


\section{Preparation of solutions \\ Diluent}

A mixture of equal volume of acetonitrile and water was prepared.

\section{Buffer preparation}

Buffer was prepared by weighing and mixing $5.70 \mathrm{~g}$ of $\beta$ Cyclodextrin and $5.60 \mathrm{~g}$ of Di-sodium hydrogen phosphate in 1000 $\mathrm{ml}$ of water. It was sonicated to dissolve and then added $8.0 \mathrm{~mL}$ of Triethylamine. Adjusted the $\mathrm{pH}$ to 7.0 with Ortho-phosphoric acid and filtered through $0.45 \mu$ filter.

\section{Mobile Phase A}

A mixture of $1000 \mathrm{~mL}$ of buffer and $150 \mathrm{~mL}$ of acetonitrile was prepared, mixed and sonicated to remove air bubbles.

\section{Mobile Phase B}

A mixture of $500 \mathrm{~mL}$ of water and $500 \mathrm{~mL}$ of acetonitrile was prepared, mixed and sonicated to remove air bubbles.

\section{Blank preparation}

Transferred $5.0 \mathrm{~mL}$ of $\mathrm{N}, \mathrm{N}$-dimethylsulphoxide into $50 \mathrm{~mL}$ volumetric flask and diluted up to the mark with diluent.

\section{Standard Stock solution}

Accurately weighed and transferred $15.0 \mathrm{mg}$ of $\mathrm{S}$ enantiomer of Tedizolid phosphate reference standard into $100 \mathrm{~mL}$ volumetric flask. $10 \mathrm{~mL}$ of $\mathrm{N}, \mathrm{N}$-dimethylsulphoxide was added and diluted up to the mark with diluent.

\section{Standard solution- $A$}

$5.0 \mathrm{~mL}$ of standard stock solution was transferred to 50 $\mathrm{mL}$ volumetric flask, $5.0 \mathrm{~mL}$ of $\mathrm{N}, \mathrm{N}$-dimethylsulphoxide was added and diluted up to the mark with diluent.

\section{Standard solution-B}

$5.0 \mathrm{~mL}$ of standard solution-A was transferred to $50 \mathrm{~mL}$ volumetric flask, added $5.0 \mathrm{~mL} \mathrm{~N}, \mathrm{~N}$-dimethylsulphoxide and diluted up to mark with diluent.

\section{System Suitability solution}

Weighed and transferred accurately about $50.0 \mathrm{mg}$ of (R)-Tedizolid phosphate reference standard in to $50 \mathrm{~mL}$ volumetric flask. First dissolved in $5 \mathrm{~mL}$ of $\mathrm{N}, \mathrm{N}$ dimethylsulphoxide. Added $5.0 \mathrm{~mL}$ of standard solution-A and diluted up to the mark with diluent.

\section{Test solution}

Weighed and transferred accurately about $50.0 \mathrm{mg}$ of sample in to $50 \mathrm{~mL}$ volumetric flask, dissolved in $5 \mathrm{~mL}$ of $\mathrm{N}, \mathrm{N}$ dimethylsulphoxide and diluted up to the mark with diluent.

\section{Procedure}

Using auto sampler injector all solutions of equal injection volume $(5 \mu \mathrm{L})$ was injected, blank preparation followed by system suitability solution, standard solution-B six replicates and test solution. In system suitability solution R-Tedizolid phosphate peak is found eluting at retention time of about 37 minutes and the relative retention time of $\mathrm{S}$-enantiomer is about 0.93. Resolution between both peaks was more than 1.5 and relative standard deviation (RSD) of peak area of six replicate injection of standard solution-B for S-enantiomer peak was found less than $5.0 \%$. S-enantiomer content in Tedizolid phosphate drug substance was calculated by external standard method.

\section{RESULTS AND DISCUSSION}

\section{Analytical method development}

Key parameters that affected the enantioseparation included the type and concentration of the chiral selector, whether an organic solvent was added or not, type of organic solvent, regarding to its protic or aprotic properties, $\mathrm{pH}$ of the mobile phase, stationary phase and column temperature. These parameters were optimized to achieve the highest enantioseparation in the Tedizolid phosphate enantiomers.

\section{Stationary phase selection}

Considering the structural similiarity of oxazolidine of linezolid, first trial was carried out as per the method of USP linezolid In-Process Revision (USP, 2014). Chiralpak AD-H, 250 $\mathrm{mm} X 4.6 \mathrm{~mm}, 5 \mu \mathrm{m}$ column consist of mobile phase Hexane : absolute alcohol : Trifluroacetic acid (650:350:1). In this method both enantiomers are resolved but recovery of S-enantiomer peak is not achieved because both peaks are asymmetrical. Different Mobile phase composition of solvents like n-Hexane, ethanol, nHeptane, isopropyl alcohol, acetonitrile and TFA (trifluoroacetic acid) trials were taken but recovery is not achieved. Solubility of Tedizolid phosphate is major constrain while trying with chiralpak ADH column. API is soluble only in N,N-dimethylsulfoxide which is not advisable to use with $\mathrm{ADH}$ column because it spoils the stationary phase. Normal phase chromatography with another chiral stationary phases were tried such as Chiralcel OD-H and Lux Amylose-2 with different solvents and modifiers including nHexane, isopropyl alcohol, ethanol , trifluroacetic acid to achieve the separation and recovery of S-isomer but no satisfactory results were found.

Further trials planned with immobilized chiral stationary phases as Chiralpak IA, ID-3 column were carried out with different composition of solvents and modifiers like ethanol, methyl tert. butyl ether, dichloromethane, trifluroacetic acid, diethylamine but resolution was not satisfactory.

Further trials planned on reverse phase chiral stationary phases like Chiralcel OD-RH, Chiralpak AGP column tried with different buffers such as ammonium carbonate, disodium hydrogen phosphate, potassium dihydrogen phosphate at $\mathrm{pH} 2.2,5.0,8.8$ with solvent composition like acetonitrile, methanol but no 
resolution found. Crown-pack CR (+) column tried with buffer as perchloric acid pH 1.0, 1.5 and 2.0 but no resolution observed.

Chiradex HR column trials taken using different buffers like ammonium formate, sodium dihydrogen phosphate, ammonium dihydrogen phosphate, potassium dihydrogen phosphate, triethylamine at $\mathrm{pH} 3.0,5.0$ and 7.0. By using buffer 25 $\mathrm{mM}$ disodium hydrogen phosphate at $\mathrm{pH} 5.0$ : acetonitrile (70:30) with flow rate $0.8 \mathrm{~mL} / \mathrm{min}$ resolution found 1.4 . Recovery was found $98.0 \%$ to $102.0 \%$ but both enantiomers are eluting very late and run time was $180 \mathrm{~min}$. Different trials taken to shorten the run time but at shorter run time resolution was reduced and recovery was not achieved.

Out of these many trials both enantiomers were found resolved only on Chiradex column which contain $\beta$-cyclodextrin. So the next trial was carried out using $\beta$-cyclodextrin additive in a buffer and acetonitrile as organic solvent with $\mathrm{C}-18$ column but this gives no resolution.

Finally desired resolution is obtained with Phenomenex Luna Phenyl-Hexyl column along with combination of additive $\beta$ Cyclodextrin, disodium hydrogen phosphate and acetonitrile. Resolution between $\mathrm{R}$ and $\mathrm{S}$-enantiomer is 1.7 and achieved base to base separation between both enantiomers. Run time was reduced to 75.0 minutes.

\section{Effect of composition of mobile phase}

The mobile phase composition was found to influence the retention time and resolution of tedizolid phosphate enantiomers. Further investigation was conducted by varying the volumetric ratio of aqueous buffer and acetonitrile under the following conditions: $\mathrm{pH}$ of 7.0, $\beta$-CD concentration of $5 \mathrm{mM}$ and disodium hydrogen phosphate concentration of $40 \mathrm{mM}$. The variation of organic modifiers' content has substantial impact on enantioseparation of Tedizolid phosphate. Upon increasing the proportion of acetonitrile, both retention time and resolution

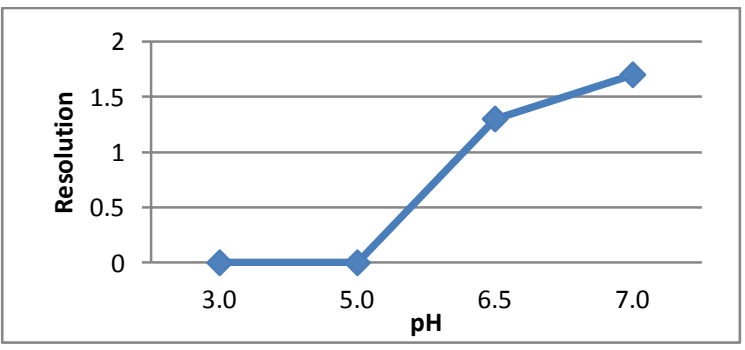

Fig. IV: Effect of $\mathrm{pH}$ on resolution of $\mathrm{R}$ and $\mathrm{S}$ enantiomers. decreased accordingly. In order to obtain satisfactory resolution and retention time, the volume ratio of mobile phase A $1000: 150$ was selected.

\section{Effect of the Concentration of $\beta$-cyclodextrin}

The effect of $\beta$-CD concentration on enantioseparation was investigated under the $\mathrm{pH}$ of 7.0. A range of $\beta-\mathrm{CD}$ concentrations from $1 \mathrm{mM}$ to $10 \mathrm{mM}$ were tested. The concentration of $\beta$-CD has a significant impact on the resolution. Resolution is getting reduced after reducing concentration to $1 \mathrm{mM}$. By increasing concentration both enantiomers are base to base resolved. Resolution was found sufficient about 1.7 at $5 \mathrm{mM}$ as well as $10 \mathrm{mM}$ concentration of $\beta$-CD. The $\beta$-CD concentration of $5 \mathrm{mM}$ was chosen as a compromise between a good resolution and a relatively short retention time.

\section{Effect of pH on resolution}

Chiral recognition is based on the formation of a stable inclusion complex and hydrogen bonding interaction with the guest enantiomer and $\mathrm{pH}$ plays an important role in maintaining the stability of the complex. The influence of mobile phase $\mathrm{pH}$ in the range of 3.0 to 7.0 with $5 \mathrm{mM}$ chiral additive and $40 \mathrm{mM}$ disodium hydrogen phosphate was investigated. As shown in figure-IV with decrease in $\mathrm{pH}$ of the mobile phase, resolution decreased.

At pH 3.0 and 5.0 both enantiomers are not separate, at $6.5 \mathrm{pH}$ resolution is 1.3 and at $7.0 \mathrm{pH}$ resolution is achieved 1.7 and peaks are base to base separate. Hence $\mathrm{pH} 7.0$ was found to be the optimum $\mathrm{pH}$ for the analysis. Along with the $\mathrm{pH}$, triethylamine and concentration of discodium hydrogen phosphate was studied. It was observed that the band broadened without triethylamine, resulting in lower resolution. One unknown impurity was eluting very closely with S-enantiomer peak which is got separated by addition of disodium hydrogen phosphate.

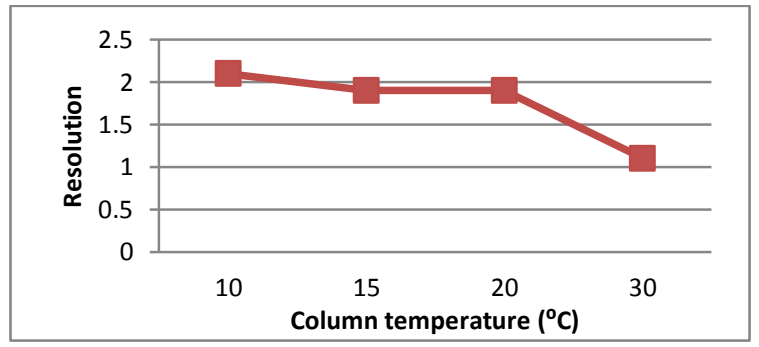

Fig. V: Effect of column temperature on resolution of R and $\mathrm{S}$ enantiomers.

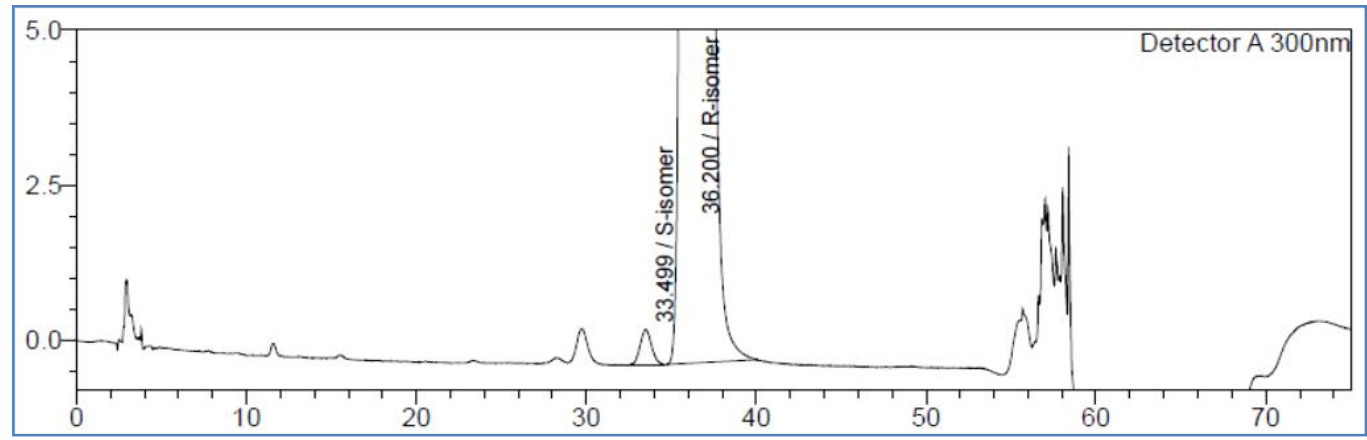

Fig. VI: System suitability solution chromatogram where R-Tedizolid phosphate is eluting at RT 36.2 minutes and S-enantiomer at relative retention time 0.93 . 


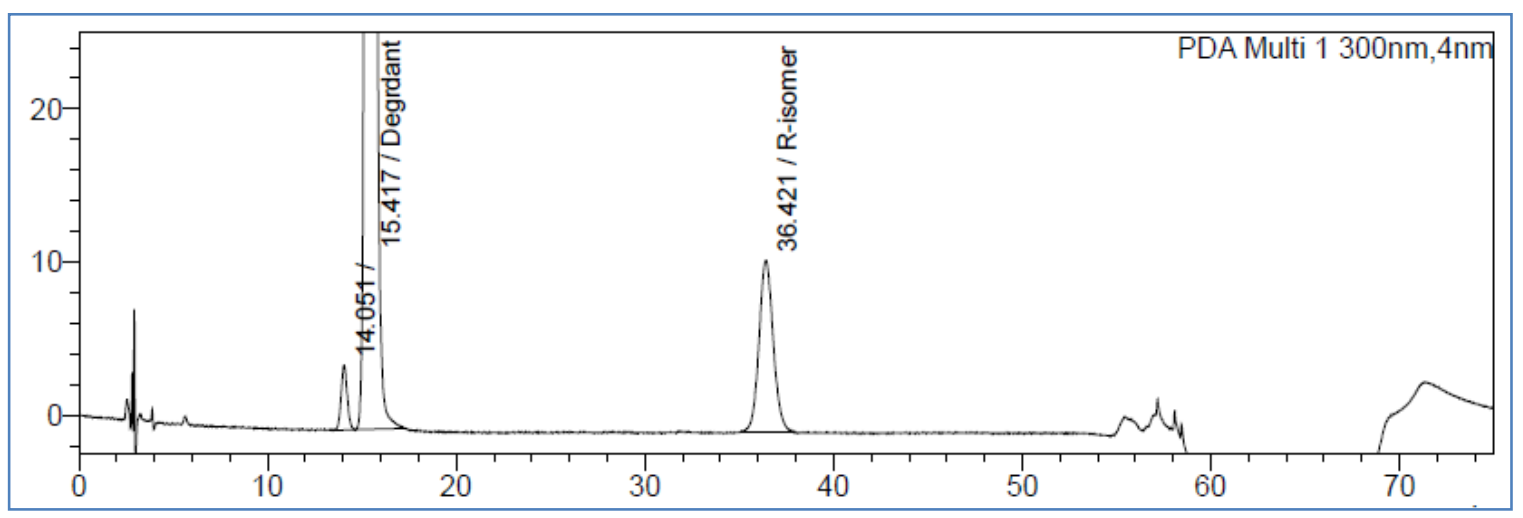

Fig. VII: Chromatogram of Tedizolid phosphate exposed to $5 \mathrm{~N}$ sodium hydroxide where major degradant is eluting at RT 15.4 minutes.

\section{Effect of the column temperature}

The effect of column temperature on the resolution of Tedizolid phospahte enantiomers was studied in a range of $10^{\circ} \mathrm{C}$ to $30^{\circ} \mathrm{C}$. As shown in figure- $\mathrm{V}$ increase in column temperature, the resolution decreased, at $30^{\circ} \mathrm{C}$ the resolution was 1.1 . Resolution increases after reducing column temperature, where at $20^{\circ} \mathrm{C}, 15^{\circ} \mathrm{C}$ and $10^{\circ} \mathrm{C}$, the resolution is $1.9,1.9$ and 2.1 respectively. By reducing temperature, resolution is increased but during long analysis sequence instrument stop due to leak detection because of water condensation in column compartment. To achieve a fair balance between higher resolutions and avoid water condensation, $20^{\circ} \mathrm{C}$ was chosen as the operating temperature.

\section{Analytical Method Validation}

The proposed method was validated through the examination of specificity, solution stability, limit of detection (LOD), limit of quantitation (LOQ), linearity, accuracy, ruggedness and robustness study as per ICH guideline.

\section{Specificity and Force degradation study}

Specificity is defined as the ability of the method to determine accurately and specifically the analyte of interest in the presence of other components in a sample matrix that may be expected to be present in the sample matrix under the stated conditions. Specificity of the method was evidenced by comparing blank, R-Tedizolid phosphate, S-Tedizolid phosphate and all specified impurities separate injections as well as spiking Senantiomer into R-Tedizolid phosphate test solution. There are no interfering peaks at the retention times of R-Tedizolid phosphate eluting at 36.2 minutes and S-enantiomer at relative retention time 0.93. Referring to figure VI of system suitability solution chromatogram The peak purity of both enantiomers is checked with photo diode array detector (PDA). It is found that peak purity index of R-Tedizolid phosphate and S-enantiomer is 1.00 and 0.99 respectively which proves that both enantiomer peaks are pure without any interference. Force degradation is performed to ensure interference of any degradant at the retention time of S-enantiomer peak. Tedizolid phosphate sample was exposed to different stress conditions and then tested using the proposed method of analysis.
The API batch was heated at $105^{\circ} \mathrm{C}$ for 24 hours and then the test solution was prepared and injected. Similarly sample was treated with base $5 \mathrm{~N}$ sodium hydroxide, with $5 \mathrm{~N}$ hydrochloric acid exposed to ultra-violet light for 24 hours and 3\% hydrogen peroxide solution 24 hours. After exposure samples were prepared separately and tested using the proposed chiral purity method with photo diode array (PDA) detector. It was observed that Tedizolid phosphate is stable when exposed to heat, UV light, acid and oxidation. In base degradation one major impurity is formed about 92\% eluting at retention time 15.4 minutes. The base degradation chromatogram is given in figure VII. On basis of forced degradation study it can be concluded that no degradant is eluting at the retention time of R and S-Tedizolid phosphate. Peak purity of both enantiomer peaks is ensured by PDA detector found passing in all degraded samples.

\section{Solution stability}

Drug stability in Active Pharmaceutical Ingredient is a function of storage conditions and chemical properties of the drug and its impurities. Conditions used in stability experiments should reflect situations likely to be encountered during actual sample handling and analysis. Stability data are required to show that the concentration and purity of analyte in the sample at the time of analysis corresponds to the concentration and purity of analyte at the time of sampling. The solution stability till twenty three hours of Tedizolid phosphate API had been checked by injecting system suitability solution. Solution was prepared fresh before injection and immediately injected and same solution was injected after twenty three hours. Tedizolid phosphate and S-enantiomer is found stable in solution form till twenty three hours.

\section{Limit of detection}

The limit of detection (LOD) is the lowest concentration of analyte in a sample that can be detected but not necessary quantified. The obtained LOD value of S-enantiomer of Tedizolid phosphate is tabulated in Table-I.

$$
\mathrm{LOD}=3.3 \times \sigma / \mathrm{S}
$$

Where, $\sigma=$ the standard deviation of the response and $\mathrm{S}=$ slope of the calibration curve 


\section{Limit of quantitation}

The limit of quantitation is the lowest concentration or amount of analyte that can be determined quantitatively within an acceptable level of repeatability precision and trueness.

Limit of quantitation $(\mathrm{LOQ})=10.0 \times \sigma / \mathrm{S}$

Where, $\sigma=$ the standard deviation of the response and $S=$ slope of the calibration curve

Precision at LOQ is confirmed by six replicate analyses of $\mathrm{S}$ enantiomer at LOQ level. LOD, LOQ and RSD at LOQ level is tabulated in Table I.

Table I: LOD, LOQ and precision.

Name of the compound $\quad$\begin{tabular}{lll}
\hline \\
S-Tedizolid phosphate
\end{tabular}

\section{Linearity}

The ability of the method to obtain test results proportional to the concentration of the analyte within a given range. It was evaluated by linear regression analysis and calculated by the least square regression method. Under the experimental conditions, the S-enantiomer peak area vs. concentration plot for the proposed method was found to be linear over the range of LOQ concentration, $25 \%, 50 \%, 80 \%, 100 \%, 120 \%$ and $150 \%$ of the specified limit $\left(0.30 \mu \mathrm{g} \mathrm{mL}^{-1}\right.$ to $\left.2.25 \mu \mathrm{g} \mathrm{mL}^{-1}\right)$ with a regression coefficient 0.998 . The regression coefficient $\left(\mathrm{r}^{2}\right)$ is $>0.99$ is generally considered as evidence of acceptable fit of the data to the regression line.

\section{Accuracy}

Accuracy can be defined as the closeness of agreement between a test result and the accepted reference value. Accuracy of the method was determined by recovery study. Analytical method may be considered validated in terms of accuracy if the mean value is within $\pm 20 \%$ of the actual value. During recovery study, Tedizolid phosphate API batch was analyzed and then $\mathrm{S}$ enantiomer of known concentration is spiked in the API at LOQ level, $50 \%, 100 \%$ and $150 \%$ with respect to the limit of Senantiomer.

Table II: Recovery study.

\begin{tabular}{ccccc}
\hline Name of the compound & $\begin{array}{c}\text { LOQ } \\
\text { level }\end{array}$ & $\begin{array}{c}\mathbf{5 0 \%} \\
\text { level }\end{array}$ & $\begin{array}{c}\mathbf{1 0 0 \%} \\
\text { level }\end{array}$ & $\begin{array}{c}\mathbf{1 5 0 \%} \\
\text { level }\end{array}$ \\
\hline S-Tedizolid phosphate & $96.9 \%$ & $100.9 \%$ & $104.3 \%$ & $105.3 .0 \%$ \\
\hline
\end{tabular}

As per ICH guideline Q3A-R2 the limit of known impurity should be $0.15 \%$ if daily maximum dose is less than $2.0 \mathrm{~g}$ (ICH, 2006). The dose of Tedizolid phosphate is $200 \mathrm{mg} /$ day with oral and parenteral route of administration (Drugs.com, 2014). So the limit of S-enantiomer as known impurity is decided $0.15 \%$. Recovery of S-enantiomer was found in the range of $80.0 \%$ to $120.0 \%$, which was well within the acceptance criteria. Results are tabulated in Table-II.

\section{Ruggedness study}

The (intra-laboratory tested) behavior of an analytical process when small changes in environment and/or operating condition are made. The ruggedness of the method was evaluated by estimating \% RSD of standard solution tested by two different analysts using different HPLC instrument and columns on different days. \% RSD of area of S-enantiomer peak on two different days is found $1.8 \%$ and $1.0 \%$ with resolution between $\mathrm{R}$ and S-enantiomers in system suitability solution is found 1.78 and 2.02 respectively. Six preparations of S-enantiomer spiked in RTedizolid phosphate was prepared by two different analysts and analyzed on two different days. The \% RSD of S-enantiomer of total twelve preparations is found $3.99 \%$ which proves that the method is rugged and delivers accurate and consistent results.

\section{Robustness}

Robustness is a measure of the capacity of the analytical procedure to remain unaffected by small but deliberate variations in method-performance parameters, which provides an indication of its reliability during normal usage. Robustness of the method was determined by analyzing the system suitability solution and batch analysis with deliberate change in the parameters like (a) flow rate of mobile phase $\pm 0.1 \mathrm{ml} / \mathrm{min}$ (b) mobile phase solvent ratio variation $\pm 10 \%$, (c) mobile phase $\mathrm{pH} \pm 0.1$ and (d) column temperature $\pm 5^{\circ} \mathrm{C}$. Results are discussed in table III.

Table III: Robustness study.

\begin{tabular}{ll}
\hline Change in the chromatographic parameter & Resolution \\
\hline Flow rate & \\
\hline $0.9 \mathrm{~mL} / \mathrm{min}$ & 1.7 \\
$1.1 \mathrm{~mL} / \mathrm{min}$ & 1.5 \\
\hline Mobile phase solvent variation & \\
\hline$+10 \%$ Acetonitrile & 1.02 \\
$-10 \%$ Acetonitrile & 1.7 \\
\hline Mobile phase $\mathbf{p H}$ & \\
\hline $\mathrm{pH} 6.9$ & 1.6 \\
pH 7.1 & 1.5 \\
\hline Column temperature & \\
\hline Temperature $15^{\circ} \mathrm{C}$ & 1.6 \\
Temperature $25^{\circ} \mathrm{C}$ & 1.5 \\
\hline
\end{tabular}

Critical parameter in the method is resolution that is studied during robustness evaluation. It had been observed that flow rate, $\mathrm{pH}$ of the mobile phase and column temperature not affecting much in day to day variation of chromatographic parameters. But the concentration of acetonitrile in the mobile phase-A has significant impact on enantioseparation.

\section{CONCLUSION}

This paper describes a new and reliable method for Tedizolid phosphate enantioseparation by HPLC, using $\beta$-CD as a 
chiral mobile phase additive. $\beta$-cyclodextrin was used for the first time as a chiral mobile phase additive for determining the enantiomeric composition of racemic Tedizolid phosphate, rather than involving costly chiral stationary phases. The RP-HPLC chiral analytical method satisfies all validation parameters like system suitability, precision, specificity, accuracy, linearity of detector response, ruggedness and robustness. It indicates that the method is stable and suitable for the quantification of $\mathrm{S}$ enantiomer of R-Tedizolid phospahte. Hence, the simple cost effective validated method can be used for routine analysis in quality control laboratories in the pharmaceutical industry.

Future plan is decided to identify the degradant impurity which is observed about $92 \%$ during force degradation study. This obvious degradant is required to synthesize or enrich for structure elucidation purpose. After quanlification of impurity standard it can be quantified in Tedizolid phosphate API either using chiral or achiral purity method.

\section{ACKNOWLEDGEMENT}

Thanks to the management of Wanbury Limited for valuable support and encouragement.

\section{Financial support and sponsorship: Nil.}

Conflict of Interests: There are no conflicts of interest.

\section{REFERENCES}

Bressolle F., Audran M., Pham T., Vallon J. Cyclodextrins and Enantiomeric Separations of Drugs by Liquid Chromatography and Capillary Electrophoresis: Basic Principles and New Developments. Journal of Chromatography B: Biomedical Sciences and Applications, 1996; 687: 303-336.

Branch SK. Guidelines from the International Conference on Harmonisation (ICH). J. Pharm Biomed Anal; 2005; 38: 798-805.

Christodoulou E. An Overview of HPLC Methods for the Enantiomer Separation of Active Pharmaceutical Ingredients in Bulk and Drug Formulations; Current Organic Chemistry; 2010; 14: 2337-2347.

Drugs.com, 2014; Tedizolid phosphate. Available at: https://www.drugs.com/dosage/tedizolid.html

Elaine W., Saba R.; Tedizolid Phosphate (Sivextro); A SecondGeneration Oxazolidinone to Treat Acute Bacterial Skin and Skin Structure Infections; Pharmacy and Therapeutics; 2014; 39(8): 555-558.

EMA. 2015. European Medicines Agency; Assessment report; Procedure No. EMEA/H/C/002846/ 0000

FDA. 2014. Food and Drug Administration; Center For Drug Evaluation And Research; Application Number: NDA 205435 CMC Review; Available at: https://www.accessdata.fda.gov/drugsatfda_docs/nda/2014/205435Orig1s0 00ChemR.pdf

FDA, 2009; U.S. Department of Health and Human Services Food and Drug Administration; Guidance for Industry ANDAs: Impurities in Drug Substances

Guan J., Yang J., Bi Y., Shi S., Yan F., Li F. Determination of the Enantiomeric Impurity in S-(-)Pantoprazole using High Performance Liquid Chromatography with Sulfobutyletherbeta-Cyclodextrin as Chiral Additive. Journal of Separation Science, 2008; 31:288-293.
Han S.; Direct Enantiomeric Separations by High Performance Liquid Chromatography Using Cyclodextrins. Biomedical Chromatography, 1997; 11: 259-271.

ICH. 2005. Validation of analytical procedures: Text and methodology Q2 (R1); International conference on harmonization of technical requirements for registration of pharmaceuticals for human use.

ICH. 2006. Impurities in new drug substances Q3A (R2); International conference on harmonization of technical requirements for registration of pharmaceuticals for human use.

Katarzyna M., Ewa G., Judyta C., El'zbieta B. Chiral Separation of Tedizolid Using Charge Single Isomer Derivatives Ofcyclodextrins By Capillary Electrokinetic Chromatography. Journal of Pharmaceutical and Biomedical Analysis, 2016; 120: 402-412.

Le'On A., Olives A., Del Castillo B., Mart'In M. Influence of the presence of Methyl Cyclodextrins in High Performance Liquid Chromatography Mobile Phases on the Separation of $\beta$-Carboline Alkaloids. Journal of Chromatography A, 2008; 1192: 254-258.

Płotka J., Biziuk M., Morrison C. Common Methods for the Chiral Determination of Amphetamine and Related Compounds; I. Gas, Liquid and Thin-Layer Chromatography. Trends in Analytical Chemistry. 2011; 30: 1139-1158.

Rauws AG, Groen K. Current Regulatory Guidance on chiral medicinal products; Canada, EEC, Japan, United States. Chirality; 1994; $6(2) ; 72-5$.

Reyes-Reyes M., Roa-Morales G., Melgar-Fern'R., ReyesP'Erez H., Balderas-Hern P. UHPLC Determination of Enantiomeric Purity of Sertraline in the Presence of its Production Impurities; Chromatographia, 2014; 77: 1315-1321.

Santini DA, Sutherland CA, Nicolau DP. Development of a High Performance Liquid Chromatography Method for the Determination of Tedizolid in Human Plasma, Human Serum, Saline and Mouse Plasma. Journal of Chromatography Separation Technique, 2015; 6; 4.

Tong S., Zhang H., Shen M., Ito Y., Yan J. Enantioseparation of Mandelic Acid Derivatives by High Performance Liquid Chromatography with Substituted $\beta$-Cyclodextrin as Chiral Mobile Phase Additive and Evaluation of Inclusion Complex Formation. Journal of Chromatography B, 2014; 962: 44-51.

Taschwer M., Seidl Y., Mohr S., Schmid A. Chiral Separation of Cathinone and Amphetamine Derivatives by HPLC/UV using Sulfated $\beta$-Cyclodextrin as Chiral Mobile Phase Additive. Chirality, 2014; 26: 411-418.

USP. 2014. 40(3) In-Process Revision: Linezolid; United State Pharmacopeia; USP38-NF33-1S.

Voon O., Shawn F., Edward F., Howard J., Jeffrey B., Kenneth B., Philippe P. Absorption, Distribution, Metabolism, And Excretion Of The Novel Antibacterial Prodrug Tedizolid Phosphate. Drug Metabolism and Disposition; 2014; 42: 1275-1284.

Xu J., Zhao W., Ning Y. et al.; Enantiomer Separation of Phenyllactic acid by HPLC with HP- $\beta$-Cyclodextrin as Chiral Mobile Phase Additive. Journal of Inclusion Phenomena and Macrocyclic Chemistry, 2013; 76: 461-465.

Xie J., Tan Q., Yang L. A Simple and Rapid Method for Chiral Separion of Amlodipine using Dual Chiral Mobile Phase Additives, Analytical Methods, 2014; 6: 4408-4413.

Ye J., Yu W., Chen G., Shen Z., Zeng S. Enantiomeric Separation of 2-Arylpropionic acid Nonsteroidal Anti-Inflammatory Drugs by HPLC with Hydroxypropyl- $\beta$-Cyclodextrin as Chiral Mobile Phase Additive. Biomedical Chromatography, 2010; 24: 799-807.

\section{How to cite this article:}

Anerao A, Dighe V, John S, Pradhan N. Enantioseparation of Tedizolid phosphate by RP-HPLC, using $\beta$-Cyclodextrin as a Chiral Mobile Phase Additive. J App Pharm Sci, 2017; 7 (10): 030036. 